\title{
El arraigo territorial en la internacionalización del vino español
}

Territorial identity in the internationalization of Spanish wine

\author{
Rogelio JORGE MARTÍN \\ Universidad Nacional de \\ Educación a Distancia (UNED). \\ Universidad de Castilla-La Mancha \\ rjorge@economistas.org \\ www.rogeliojorge.es
}

\begin{abstract}
Resumen: El principal problema exportador que se encuentra el sector del vino en España es la falta de valores intangibles que lo pongan en valor. Con esto nos referimos a una historia, un territorio y una cultura que hagan distintivo al producto. En este artículo analizamos el panorama general de la internacionalización del vino español, el nuevo marketing del vino y las bases del arraigo territorial que lo hacen posible. Para este último aspecto recurriremos a una fuente histórica tan valiosa como son Las Relaciones de Felipe II. Finalmente estudiaremos el caso de la denominación de origen el Bierzo, como ejemplo exitoso de aplicación de este modelo de marketing territorial.
\end{abstract}

Abstract: The main export problem found in the wine sector in Spain is the lack of intangible assets from which wine derives value. These refer to a history, a territory and a culture that bestow the product its distinctive qualities. In this article we examine the general panorama of internationalization of Spanish wine, the new marketing of wine and the territorial bases that make it possible. Concerning this last aspect, we will draw lessons from a historical tool as valuable as Las Relaciones of Philip II. To sum up, we will study the Designation of Origin el Bierzo, a successful case of the application of this territorial marketing model.

Palabras clave: vino, exportación, territorio, internacionalización, marketing internacional, marketing mix, Bierzo, enoturismo, Felipe II, denominación de origen.

Keywords: wine, export, territory, internationalization, international marketing, marketing mix, Bierzo, wine tourism, Philip II of Spain, designation of origin. 


\section{Sumario:}

\section{Introducción.}

II. La internacionalización del vino español.

2.1. El precio medio del vino español.

2.2. La diversificación de mercados internacionales.

2.3. El plan de internacionalización y su importancia.

2.4. Elegir el mejor camino a la internacionalización.

III. Determinantes en la internacionalización del vino.

IV. El arraigo territorial: el vino en Las Relaciones de Felipe II.

4.1. Descripción de los pueblos de España en la segunda mitad del siglo XVI.

4.2. Respuestas vinculadas al comercio del vino.

4.3. Respuestas vinculadas a la extensión del viñedo y a la producción.

4.4. Respuestas vinculadas a la tierra o a la propiedad.

V. El Bierzo: paradigma de marketing territorial.

5.1. Inversiones de iniciativa privada entorno al vino en la comarca.

5.2. Enólogos de prestigio.

5.3. El turismo y la promoción entorno al vino.

5.4. El vino: elemento de fijación de población.

5.5. Denominación de origen de vino Bierzo: claves en la Internacionalización.

5.6. La trazabilidad y lo autóctono.

\section{Conclusiones.}

VII. Bibliografía.

VIII. Anexo 1. Cuestionario de Las Relaciones.

Recibido: noviembre 2019.

Aceptado: enero 2020. 


\section{INTRODUCCIÓN}

Hoy en día, gran parte del vino que se produce en España -principal productor mundial de vino- se exporta a países de nuestro entorno y a precios bajos. Hecho que está ocurriendo desde hace décadas. Nuevos indicadores socio-económicos inciden en vender mejor y mayor diversificación de mercados de destino para sostenibilidad del sector.

Conseguir estos objetivos requiere de adecuada planificación en la internacionalización y saber conectar con el consumidor internacional. Éste asocia el vino a una cultura, una tierra y a una experiencia, y además de unos atributos tangibles e intangibles (carácter propio).

La geohistoria -paisaje y clima, costumbres y evolución social-, variedades autóctonas e identidad de un territorio (terruño), son parte intrínseca en la tendencia actual en el consumo del vino. En este artículo se realiza un acercamiento a la obra estadística -en un periodo anterior a esta ciencia, pero con un método muy similar- de Las Relaciones de Felipe II (segunda mitad del siglo XVI) para poner las bases del arraigo territorial vinícola, relacionándolo con algunas de las actuales denominaciones de origen (D.O.). Además, se analiza el caso de la D.O. Bierzo y los proyectos que se están llevando a cabo allí en este sentido de asociar el vino a atributos intangibles que le aportan valor.

\section{LA INTERNACIONALIZACIÓN DEL VINO ESPAÑOL}

Según el Observatorio Español del Mercado del Vino, España se ha convertido en los últimos 10 años en el mayor exportador mundial de vino en volumen, registrando un crecimiento del $50 \%$ en ese periodo. En las últimas dos décadas la mayor parte de lo producido se vende, con precio medio bajo, a países de nuestro entorno. Los vinos españoles se destinan principalmente a la Unión Europea con un 78\% del volumen total, y representando un $63 \%$ de la facturación de los vinos vendidos fuera de nuestro país ${ }^{1}$.

${ }^{1}$ OBSERVATORIO ESPAÑOL DEL MERCADO DEL VINO. «10 años de evolución del vino en el mundo y en España», 2019. 
El primer problema estratégico de la exportación vitivinícola española es la comercialización. En concreto la concentración del grueso de las ventas dentro de la Unión Europea y el bajo precio medio del vino español. Las magnitudes precio y destino de las exportaciones de vino español, son cruciales en los nuevos indicadores socio-económicos a la hora de buscar sostenibilidad en el sector del vino.

A finales de 2017 la Comisión Europea publicó su informe anual recogiendo sus proyecciones sobre la evolución de la agricultura europea hasta el año 2030, incluyendo el vino. Respecto al vino este documento pronostica la evolución tanto del consumo interno europeo como de la exportación a países fuera de la Unión Europea. Sobre el consumo interno prevé un descenso moderado y en cuanto a la venta fuera de la Unión Europea pronostica un incremento anual del $1,7 \%$ lideradas por una mayor demanda de producto embotellado ${ }^{2}$.

Rafael del Rey Salgado, en la obra La economía del vino en España y en el mundo expone que además de la innovación (productos adaptados al consumidor, envases y tangibles en general), entre los grandes retos a los que se enfrenta el sector español del vino es el de la mejor internacionalización, prevaleciendo la puesta en valor por encima del aumento de volúmenes ${ }^{3}$.

\subsection{El precio medio del vino español}

La evolución de los precios medios de los vinos españoles en las últimas dos décadas con respecto a otros países ha sido negativa. Los 11 países que lideran el ranking mundial de productores son: Francia, Italia, España, Australia, Chile, Estados Unidos, Alemania, Nueva Zelanda, Portugal, Argentina y Sudáfrica. En el año 2000 el precio medio del total de las exportaciones de cada país, medido en $€ /$ litro, situaba a España con un precio medio de 1,41€/litro, mientras que Italia vendía a 1,40 €/litro. Ambos países eran líderes mundiales (y lo siguen siendo) en producción, pero se encontraban a la cola en cuanto al precio medio, que entre los once países mencionados se situaba en $2,24 € /$ litro ${ }^{4}$.

${ }^{2}$ EUROPEAN COMMISSION, «EU Agricultural Outlook for the agricultural markets and income 2017-2030», 2017. 10 de noviembre de 2019.

$<$ https://ec.europa.eu/agriculture/sites/agriculture/files/markets-and-prices/medium-termoutlook/2017/2017-fullrep_en.pdf $>$.

${ }^{3}$ DEL REY SALGADO, R., «Retos de futuro del sector vitivinícola en España». La economía del vino en España y en el mundo, 2014, pp. 667-710.

${ }^{4}$ OBSERVATORIO ESPAÑOL DEL MERCADO DEL VINO, «Principales exportadores mundiales de vino - marzo 2019», 10 de noviembre de 2019. <https://www.oemv.es/principalesexportadores-mundiales-de-vino-marzo-2019> 
En marzo de 2019 la radiografía muestra que el precio medio del vino español es de $1,42 € /$ litro, es decir, prácticamente el mismo precio que dos décadas antes. Otros países del entorno europeo sí han visto incrementado el precio medio de venta. En el caso de Italia y Francia (productores parecidos a España) los precios medios son de 3,11 €/litro para el vino italiano y de $6,74 € /$ litro para el vino francés, duplicando el precio que tenían en el año 2000. Si se contempla solo el precio medio de vino envasado, España mejoraría un puesto en este ranking de precios medios de vino envasado, aunque estaría todavía en posiciones muy bajas, solamente delante de Portugal y Sudáfrica 5 .

\subsection{La diversificación de mercados internacionales}

La diversificación de mercados internacionales es una labor que conlleva, en la mayoría de los casos, una inversión de recursos tanto económicos como humanos que en muchos casos no son fácilmente asumibles por el exportador español.

Los beneficios de un mayor grado de diversificación de mercados son superiores a los inconvenientes. Por ejemplo, la inestabilidad económica de ciertas áreas geográficas, la volatilidad en las divisas, barreras arancelarias y legales, cuestiones geopolíticas, la competencia de países productores emergentes, el ciclo de vida del producto, así como el cobro, hacen que la diversificación sea aconsejable e incluso necesaria en muchos casos.

El informe de la Dirección Territorial de Comercio y del Instituto de Comercio Exterior (ICEX) para Castilla-La Mancha de 2019 indica, en su epígrafe "Perfil general de la empresa exportadora", la existencia de una dualidad en las empresas que exportan de forma regular. Coexiste un elevado número de empresas que exportan poco y un número reducido de empresas que concentran el grueso de la exportación. Según este informe solo en torno a 7.000 empresas de todo el territorio nacional, incluyendo a todos los sectores, no solo el vino, exportaron el $95 \%$ del total. Este dato constata asimismo una correlación positiva entre el tamaño de las empresas y la exportación continuada. Por lo tanto, el éxito y la consolidación en los mercados internacionales depende, generalmente, del tamaño de las mismas ${ }^{6}$.

5 Ídem.

6 ICEX, La Economía Internacional de Castilla-La Mancha, 2018-2019. Dirección Territorial de Comercio / ICEX en Castilla-La Mancha Secretaría de Estado de Comercio, Toledo 2019. 27 de junio de 2019, <www.icex.es>. 
Sin embargo, el caso del vino es especial. El consumidor internacional de vino, aquel al que pretenden llegar las bodegas españolas, independientemente de su tamaño, asocia el vino a unos atributos intangibles (una cultura, una tierra y a una experiencia), además de a unos atributos tangibles (envase, etiquetado, etcétera). Por lo tanto, la empresa que ofrezca algunos de estos atributos intangibles y los sepa comunicar, tiene una oportunidad clara para abrirse camino en nuevos mercados y canales de venta. Es importante remarcar que el éxito no estaría necesariamente ligado al tamaño de la empresa exportadora.

Además, los vinos con Denominación de Origen Protegida (DOP), con Indicación Geográfica Protegida (IGP) o aquellos en los que se indique la variedad de uva de vinificación pueden beneficiarse de la concesión de ayudas para medidas de promoción o información de los vinos de la Unión Europea en terceros países a través de las ayudas OCM (Organización Común de Mercado) para el vino. De esta manera desde la Unión Europea a través del Ministerio de Agricultura y Pesca, Alimentación y Medio Ambiente se apoya la promoción en mercados de terceros países para el periodo 2019/2023, o lo que es lo mismo, diversificación geográfica de venta de vino fuera de la $\mathrm{UE}^{7}$. El acceso a estos programas requiere de una tramitación previa de varios meses. Por lo tanto, es vital que la bodega tenga un proyecto de acción anual y haya incluido previamente sus futuras actividades de promoción del vino en su plan de internacionalización.

\subsection{El plan de internacionalización y su importancia}

En el éxito exportador hay que prestar especial atención a tres factores: entender el país de destino culturalmente, conocer los términos legales que afectan al producto y adaptación del producto al consumidor internacional.

Existen diversas razones para la internacionalización de las bodegas, pero enfocar el proceso proactivamente es clave. De acuerdo con las capacidades de la empresa (fortalezas / debilidades) y la situación del entorno y la competencia internacional (amenazas / oportunidades), se elabora un programa o plan de acción. Este se elabora con los instrumentos del marketing-mix (política de precios, producto, distribución y promoción) aplicados a los mercados internacionales que se desea actuar ${ }^{8}$.

\footnotetext{
${ }^{7}$ MINISTERIO DE AGRICULTURA, PESCA Y ALIMENTACIÓN, consultado el 10 de noviembre de 2019. <https://www.mapa.gob.es/es/agricultura/temas/regulacion-de-los-mercados/ organizaciones-comunes-de-mercado-y-regimenes-de-ayuda/ocm-vitivinicola/programa-apoyo.aspx $>$.

${ }^{8}$ CARNICER SOSPEDRA, D., «Plan de internacionalización de empresas», Valencia, Centros Europeos de Empresas Innovadoras de la Comunidad Valenciana (CEEI CV), 2011
} 
La variable precio es la única variable capaz de generar directamente ingresos para la empresa, por lo tanto, se le debe prestar especial atención ${ }^{9}$. Para establecer el precio de exportación existen diferentes determinantes relacionados con los mercados exteriores, con el ciclo de vida del producto y otras variables que son controlables por la empresa (coste, objetivo y marketing-mix). De estas variables controladas por la empresa, el coste incluye el producto en sí y todos los demás costes directos e indirectos derivados de la adaptación al mercado internacional, etcétera ${ }^{10}$. El objetivo depende de la política de precio de la empresa en un momento concreto. Finalmente, en la variable precio dentro del marketing-mix se basa en una promoción, una buena adaptación al mercado o en buscar canales más cortos y directos.

\subsection{Elegir el mejor camino a la internacionalización}

La decisión de acceder a los mercados internacionales de forma directa, indirecta o quizás compartida conlleva evaluar todas las condiciones del destino, de nuestro producto y competencia, así como la política de empresa y recursos que disponga.

Ante una limitación de recursos y un deseo de implantación directa, las bodegas deben determinar en qué mercados se quieren implantar. Una vez decidido esto, se deberá planificar una estrategia que permita mantener y consolidar la presencia en dichos mercados en función de las características particulares de cada uno de ellos. Esto no se puede hacer en base a percepciones subjetivas.

Entre las opciones posibles son: optar por un agente o agencia multicartera, por un distribuidor o por las empresas de trading. Para optar por la utilización de un agente o agencia, la política ha de centrarse en la marca, invirtiendo recursos en cada cliente captado y estableciendo estrategias de seguimiento y fidelización. Si la bodega elige utilizar un distribuidor, es conveniente establecer una estrategia colaborativa con el fin de mantener una imagen bien diferenciada frente al cliente final. Se desarrollarán acciones proactivas de promoción conjuntas que quizás impliquen un menor descuento para el distribuidor ${ }^{11}$. Finalmente, hay que señalar que las empresas de trading se enfocan más a ventas puntuales, con un escaso nivel de penetración.

\footnotetext{
${ }^{9}$ REINA PAZ, M., Marketing: qué, cuándo, cómo y dónde, Editorial Sanz y Torres, 2017, p. 123.

${ }^{10}$ CARNICER SOSPEDRA, D., "Price in international marketing », Madrid, ESIC Editorial, 2016.

${ }^{11}$ CARNICER SOSPEDRA, D., «Cómo vender en el exterior», Valencia, Centros Europeos de Empresas Innovadoras de la Comunidad Valenciana (CEEI CV), 2014.
} 


\section{DETERMINANTES EN LA INTERNACIONALIZACIÓN DEL VINO}

Los exportadores españoles en muchas ocasiones dan por hecho ciertas evidencias o referencias geográficas, históricas o culturales asociadas a sus vinos que para el consumidor internacional no siempre son tan claras. Son frecuentes afirmaciones como la siguiente: "Está claro que siempre, desde tiempos incluso desde los romanos [...] ha habido grandes exportadores españoles de vino" ${ }^{\prime 2}$. En parte, por este tipo de obviedad es objetivo del presente artículo sentar las bases empíricas del arraigo territorial del vino.

Un buen conocimiento del espacio geográfico con base territorial, paisajística y social, pueden llegar a complementar los enfoques empresariales de carácter económico del sector vitivinícola. En definitiva, el terroir o terruño es un concepto que forma parte del vino.

La geohistoria permite aportar además unas premisas esenciales para la interpretación correcta del territorio basadas en un carácter espacio-temporal, válido para articular un marco teórico que se expondrá más adelante con ayuda de Las Relaciones de Felipe II. El conocimiento de las fuentes históricas sirve como base para una correcta orientación e interpretación territorial, así como la comprensión de la relación entre los diferentes elementos que intervienen en la elaboración, comercialización y consumo del vino.

Tanto si lo que se pretende es obtener un mayor precio por los mismos como si lo que se desea es acceder a nuevos mercados internacionales o canales concretos de venta, hay que demostrar ese arraigo territorial con argumentos reales y coherentes para generar confianza entre bodega y consumidor. En definitiva, enriquecer el producto y la percepción hacia él y la marca durante el proceso de compra y de consumo, es decir, crear experiencias.

\section{EL ARRAIGO TERRITORIAL: EL VINO EN LAS RELACIONES DE FELIPE II}

El vino europeo, y concretamente el vino español, se justifica por una calidad respaldada por su origen geográfico y tradición histórica. José Luis Sánchez Hernández, en su capítulo dedicado al valor social y territorial del vino en España, deja claro que: "En torno a la viña y el vino se ha generado, históricamente, una red de agentes, recursos, prácticas, instituciones y paisajes cuya riqueza y complejidad no ha hecho sino crecer y difundirse en las

12 DEL REY SALGADO, R., «Retos de futuro del sector vitivinícola en España», $L a$ economía del vino en España y en el mundo, 2014, p. 689. 
décadas recientes"13. Una vez realizado el estudio de Las Relaciones, fuente histórico- descriptiva que puede servir como eje en el proceso de generación de valor añadido del arraigo territorial vinícola, se obtienen los siguientes resultados.

\subsection{Descripción de los pueblos de España en la segunda mitad del siglo XVI}

La obra Las Relaciones es un conjunto de preguntas y respuestas sobre geografía, historia, economía, religión, demografía, cultura, costumbres, etcétera, promovida por Felipe II en la segunda mitad del siglo XVI. Para su elaboración se diseñaron dos cuestionarios en los años 1575 y 1578, a los cuales responderían un total de 721 pueblos y ciudades. Los manuscritos originales se encuentran en la Real Biblioteca del Monasterio de San Lorenzo del Escorial ${ }^{14}$.

Entre las denominaciones que se les han dado a estos manuscritos se encuentran: Relaciones Topográficas; Descripción de los Pueblos de España; Descripción Histórica, Estadística y Geográfica; Censo General de España, etcétera. En este artículo, siguiendo a Campos y Fernández de Sevilla, se las va a denominar Las Relaciones de Felipe II.

Como herramienta para las bodegas españolas, útil en su marketing y comercialización, u otros grupos de interés, se ha realizado una primera selección de 88 respuestas de Las Relaciones, unas vinculadas al comercio interterritorial y la producción del vino y otras relacionadas con el territorio, de carácter geohistórico, social y económico. En total representan a 84 pueblos distribuidos entre 7 de las actuales Comunidades Autónomas:

\subsection{Respuestas vinculadas al comercio del vino ${ }^{15}$}

a) Venta, precio y reputación del vino

- “... se coge vino para esta villa $[\ldots]$ entra forastero y se vende...”. Iniesta (Cuenca), 26.

${ }^{13}$ SÁNCHEZ HERNÁNDEZ, J. L., «El valor social y territorial del vino en España», $L a$ economía del vino en España y en el mundo, 2014, pp. 31-66.

${ }^{14}$ CAMPOS Y FERNÁNDEZ DE SEVILLA, F. J., La Mentalidad en Castilla la Nueva en el siglo XVI. Religión, Economía y Sociedad, según las "Relaciones Topográficas" de Felipe II,San Lorenzo del Escorial 1986.

${ }^{15}$ CAMPOS Y FERNÁNDEZ DE SEVILLA, F. J., «La vid y el vino en Castilla La Nueva según las Relaciones topográficas de Felipe II»), en Cuadernos de Estudios Manchegos, 27 (2003) 39-63. 
- "... se coge cantidad de vino y mui bueno, y por tener tan buenas bodegas se detiene más tiempo que en otro ningún pueblo de su Comarca, y así ordinariamente tiene más subido precio...". Tendilla (Guadalajara), 23.

- “...hay viñas buenas...”. Villamanta (Madrid), 26.

- “...vino, mui bueno y delicado [...] se llevan a la Corte..." Mondejar (Guadalajara), 26.

- “...el vino es de tal calidad y fama que se busca y vienen por ello desde la corte de Su magestad y dende Toledo y Sevilla y otras partes por ser el vino tan bueno y de mucha calidad...". Estremera (Madrid), 35.

\section{b) Tipo de vino}

- “...los vinos que comúnmente se hacen en la dicha villa son tintos, los mejores que se cogen en las comarcas, ni en mucha parte y son afamados en muchas partes...". La Solana (Ciudad Real), 26.

- "...cógese vino tinto y poco blanco, y bueno lo tinto y lo aloque que provee la corte de ello y de muchos lugares de la comarca...". Tarancón (Cuenca), 26.

- “...hay muchos vinos tintos y blancos, y los más de ellos nuevos y de buenos que se hallan en esta comarca y destrito, especialmente los tintos, por haber muy buenas tierras para los vinos tintos...". Perales (Madrid), 23.

- “...se coge vino tinto aragonés abundantemente...”. La Depernada (Madrid), 23.

c) Procedencia de vino y otros productos agroalimentarios ${ }^{16}$

- “...es tierra en que se coge cantidad de vino [...] lo que se tiene más necesidad en la dicha villa es de aceite y pescado, y se proveen de ello de trajineros que lo vienen a vender del Andalucía y de la costa de Málaga y Sevilla". Alhambra, 26.

- “...se proveen y sustentan es de trigo, cebada y vino [...] de lo que más necesidad tiene esta villa es de aceite y pescados y frutas secas y verdes y paños, lo cual se proveen de aceite de Andalucía; frutas, de Beas y reino de Murcia; pescados, de Málaga, Almería [y] Sevilla; paños, de Segovia...”. Montiel, 26.

- "...esta villa es falta, de vino que no se coge en ella casi ninguno y se provee de La Mancha de vino, especialmente de Daimiel y Valdepeñas y el Moral...". Puertollano, 26.

${ }^{16}$ CAMPOS Y FERNÁNDEZ DE SEVILLA, F. J., Los pueblos de Ciudad Real en las Relaciones Topográficas de Felipe II, Ciudad Real, Diputación de Ciudad Real, 2009. 
- “...lo que más falta tiene esta villa comúnmente es aceite, pescados, y sal, y esto se provee de Andalucía, y ansimismo tiene falta de vino, provéese de La Mancha...". Villamayor de Calatrava, 26.

- “...se tiene más necesidad es de pan y vino, y así se proveen siempre los vecinos de la dicha villa del dicho pan y vino que falta de la villa de Valdepeñas [...] y de otras partes donde más barato la hallan". "La dicha villa es del señor don Álvaro de Bazán, marqués de Santa Cruz...”. “... dicho señor marqués de Santa Cruz labra de presente unas casas principales con cuatro torres y dicen los maestros que las labran que van a uso de Génova porque los maestros son genoveses". El Viso del Marqués, 26, 7, 36.

- “...es tierra flaca y miserable [...]. La falta que más se siente es de vino que no se coge sino muy poco, y es tierra pasajera donde todo se gasta y por ello van a La Mancha [...] y llevan de aquí de lo que aquí se coge que es miel y cera...". Saceruela, 26.

- “...necesidad de mulas que las traen de Castilla, y las venden muy caras a los labradores [...] se han criado algunas en esta villa en los años que $\mathrm{Su}$ Majestad ha dado licencia para echar el asno garañón a las yeguas... necesidad de aceite que se trae del Andalucía, que aunque se han puesto algunos olivares en esta villa [...] tiene necesidad de pescados; por estar lejos de la mar esta villa se come en ella caro y malo". Daimiel, 26.

- “...cógese [...] vino y aceituna [...] que vale la renta Su Majestad y Mesa Maestral [...] de vino que vale la renta más de trescientos mil... “. Campo de Criptana, 26.

- "...los alcaldes ni regidores ni otros oficios de la villa no tienen salarios ni otros aprovechamientos algunos, sino los dichos alcaldes sus derechos de sentencias y firmas, y los alguaciles sus derechos de ejecuciones y carcelajes y otras conforme al arancel de estos reinos". Argamasilla de Alba, 44.

- “...es tierra fría en tiempo de invierno y muy calurosa en el tiempo de verano, y no es tierra enferma y que es muy llana.". Tomelloso, 17, 18.

- “...y las cosas que en ella más se cogen de ordinario es pan, algunas frutas $\mathrm{y}$ vino $[\ldots]$ y las cosas que tiene falta son generalmente de todas, excepto de pan y vino y carne... [...] de las cosas que carece se trae: aceite del Andalucía y pescado de Sevilla, Cartagena y Málaga; hierro para las labores que es más necesario, de Vizcaya...”. Manzanares, 26.

- "Es aparejada para todo género de pan [...] y lo mismo para vino... [...]. Hay la más falta de pescados provéese de pescados de Galicia y Sevilla, y sal se trae de Villena y de otras partes; aceite, de la Andalucía.". Malagón, 26. 
4.3. Respuestas vinculadas a la extensión del viñedo y a la producción ${ }^{17}$

a) Extensión del viñedo

- “...tienen muy buenas viñas y muchas...”. Navalcarnero (Madrid), 26.

- “...lo mas de él es plantado de viñas e olivas...”. Ciruelos (Toledo), 26.

- “...tierra de labor principalmente de viñas...”. Nambroca (Toledo), 26.

- “...los llanos de la Alcarria son fríos, están plantados gran parte de ellos de viñas...". Irueste (Guadalajara), 26.

- “...hay muchas viñas a la redonda de pueblo". Daimiel (Ciudad Real), 26.

b) Producción de vino

- “...no hay otra labranza principal de que se pueda hacer cuenta sino es de las viñas...". Burguillos (Toledo), 26.

- “...se coge [...] mucho vino en grande abundancia, porque esta villa y Tordelaguna se reputa una bodega que tiene mucho nombre en el Reino...". Uceda (Guadalajara), 23.

- "...solamente tiene abundancia de pan y vino de que hay buenos años...". Daimiel (Ciudad Real), 26.

c) Producción para autoabastecimiento de la localidad

- “...se coxe algun vino para abastecimiento...”. Marjaliza (Toledo), 26.

- “...dan vino suficiente ...”. Almodóvar del Campo (Ciudad Real), 26.

- “...vino medianamente, compite con Yllana...”. Albalate de Zorita (Guadalajara), 26.

- “...en ella más se coge es pan e vino, esto moderadamente...”. Arganda (Madrid), 26.

d) Localidades con escasa producción de vino

- “...vecinos del dicho lugar cogen mui poco vino...”. Yeles (Toledo), 26

- "Recogen un poco de vino...". Hueva (Guadalajara), 26.

17 CAMPOS Y FERNÁNDEZ DE SEVILLA, F. J., «La vid y el vino en Castilla La Nueva según las Relaciones topográficas de Felipe II», en Cuadernos de Estudios Manchegos, 27 (2003) 39-63. 
- “...el vino y aceite se coge poco...”. Carabaña (Madrid), 26.

- "Se coge poco vino". Tribaldos (Cuenca), 26.

- “... hay viñas, aunque no en mucha cuantidad...”. Totanes (Toledo), 26.

e) Respuestas a causas de la baja producción

- “...es muy poco el que se coge, y cada día va en disminución a causa de un gusano que llaman arrevolvedor que ha dado en las viñas de doce años a esta parte...". Torrubia del Campo (Cuenca), 26.

- “...se coge pan y vino medianamente, y se recogiera mucho más en grande abundancia...si la caza del bosque del Pardo de Su majestad no se lo comiese y destruyese...". Aravaca (Madrid), 26.

\section{f) La falta de vino considera su mayor necesidad}

- “...hay gran falta de vino, porque no hay viñas que sean bastantes para lo provisión de este lugar...”. Azaña (Toledo), 26.

- “...la mayor necesidad este pueblo tiene es de vino...". Argamasilla de Alba (Ciudad Real), 26.

- “...de lo que más necesidad hay en este lugar es de vino....". Torrubia del Campo (Cuenca), 26.

g) Afirmaciones varias según Juan Ortega Rubio en la obra "Relaciones Topográficas de los Pueblos de España",18

- “...tierra de pan llevar, de azafrán y de vino...”. Carrascosa del Campo (Cuenca), 35.

- “...se coge miel y cera”. “...osos y lobos muchos”. Helechosa (Badajoz), 18,22 .

- “...se coge algún trigo, vino y aceite...”. Orcera (Jaén), 26.

- "Es villa del reino de Murcia". "Una fuente surte el pueblo y riega la huerta y viñas". Jumilla (Murcia), 5, 21.

- "En las huertas de regadío se coge hortaliza". "En el campo, cereales y vino...". La Alberca (Salamanca), 21, 24.

18 ORTEGA RUBIO, J., Relaciones topográficas de los pueblos de España, Madrid, Sociedad Española de Artes Gráficas, 1918. 
- “...es el primer lugar de Extremadura y su Majestad tiene en él puerto...". "...naranjos, limos, cidras [...] muy buenas granadas". "...mucha y excelente seda". Abadía (Cáceres), 3, 6, 8, 14.

- “...de pan llevar y de vino...". "Pertenece El Peral al reino de Toledo y a la Mancha que dicen de Aragón". El Peral (Cuenca), 1, 2, 3, 4, 17, 18.

- "Cógese trigo, cebada y vino". "El Júcar corre a 2 leguas de la villa". "Fue aldea de la villa de Alarcón y la Reina Católica la eximió y la hizo villa...". Villanueva de la Jara (Cuenca), 1, 2, 20, 26.

- “...conquistó Jaime I de Aragón [...] situada en la frontera del reino de Valencia...". "Cógese [...] regular cantidad de vino". Sax (Alicante), 1-5, 26.

- "Los productos mejores de la tierra son los espárragos, la miel, el vino, el queso y los melones". “...se fundó -según tradición- por los templarios". "El Tajo pasa de la villa a un cuarto de legua y en sus huertas abunda la fruta, hortalizas y legumbres". "La villa tiene un mercado todos los jueves...". Puebla de Montalbán (Toledo), 1, 2, 20, 21, 46, 55.

- “...Es tierra de labranza. “...Algunos vecinos se ocupan en hacer cántaros para agua, que son buenos y se venden bien". Puebla de Almenara (Cuenca), 23,31 .

- "Se coge trigo [...] alguna uva; de todo un poco". Hornos (Jaén), 26

- “...en el término hay cantidad grande de viñas, olivos y almendros...." "El Guadarrama corre a la distancia de legua y media; el Tajo a la de 5 y el Alberche a la de 4. En las huertas se cultivan hortalizas". "Está fundado el pueblo en dos valles y dos laderas". "En el lugar hay 370 vecinos; además 14 casas de moriscos procedentes del reino de Granada y que el Rey mandó repartir a Camarasa”. Camarena (Toledo) 20, 21, 32, 38, 39, 45.

- "Cógese trigo y cebada en su pequeño término, también vino...". "A la distancia de 2 leguas pasa el río Júcar". Quintanar del Rey (Cuenca) 20,26.

- "Población la más antigua de toda la comarca...". "Tierra de labranza...". "Situada en el distrito de Granada...". "Está en la ribera del Tajo y a media legua corren los ríos Cedena y Pusa". "En la ribera del Tajo se admira grande y fuerte castillo..." Malpica (Toledo), 2, 3, 4, 9, 20, 26, 33, 35, 36.

- "...carece de leña, hasta el punto que sus vecinos, solo queman sarmientos de las viñas...". "Tierra de labranza y de vino...". Puebla de Almoradier (Toledo), 7,18, 19, 26.

- "Pertenece al reino de Castilla la Nueva y a la Mancha de Aragón". "Tierra fría, sana y con poca leña...". "Es de poca labranza y pocos ganados, siendo su mejor granjería el vino". Provencio está situado en una llanura, y llama la atención una fortaleza antigua...". El Provencio (Cuenca), 5, 17,18,23, 28, 29, 30. 
- "En cuanto a la agricultura, se coge trigo, cebada y centeno, algo de vino...". Méntrida (Toledo), 26.

- "A doce o quince pasos de Alcalá corre el Júcar, que riega muchas tierras con árboles, viñas, etc.”. “Tierra de pan llevar...”. Alcalá del Júcar (Albacete), 20, 23.

- “...en el camino que va desde Cartagena, Murcia y Valencia a Toledo y Castilla la Vieja, había un castillo muy fuerte en medio del despoblado...". "Tierra llana, rasa y con leña suficiente de romero y de atocha. Acabóse [...] hace unos 40 las cebras". "...a una legua de la villa, hacia el Saliente y Norte, hay un camino que llaman murciano, a modo de calzada, que viene desde Cartagena y pasa por Castilla la Vieja; dícese que lo hicieron los romanos, cuando se apoderaron de España”. La Roda (Albacete), 1, 2, $3,4,17,18,57$.

- “...se halla en el reino de Murcia...". "Celebra mercado franco los jueves de cada semana". Villena (Alicante), 3, 4, 5, 6, 57.

- “...en las riberas del Tajo [...] maduran las uvas veinte días antes que en otras partes... ". Almonacid de Zorita (Guadalajara), 20,21.

- “En el campo sólo se cogen cereales y vino....". Las Pedroñeras (Cuenca), 26.

- "...radica en la Mancha de Aragón [...] a ella llegaban los reinos de Granada y de Murcia". "Tiene un mercado franco todos los martes de cada semana". "Hay mucha coscoja [...] se suele criar mucha grana, obteniendo las gentes bastante provecho; pero habiendo llegado la cochinilla de las Indias, ha bajado considerablemente el precio de dicha grana [...] había muchas cebras [...] corrían tanto, que no había caballo que las alcanzara". "Esta en el camino que va de Toledo a Murcia y a Cartagena, y en el que va desde Valencia a Sevilla". Chinchilla de Montearagón (Albacete), 2, 3, 4, 18, 54, 55.

- "...gran cantidad de aceituna y también de vino...". "...Mercado franco todos los jueves de año...". Ocaña (Toledo), 26, 46.

- "Lo que más se coge es vino...". San Clemente (Cuenca), 26.

- "Lo que en esta villa se labra mejor que en otras es el barro vidriado blanco y azulexos y otras cosas desta labor, de que se provee el Reino y parte de Portugal y las Indias...". Talavera de la Reina (Toledo), 62.

- "La villa de Yecla es paso, puerto y aduana antigua para entre estos reinos de Castilla, y el de Valencia y Aragón y otros extraños...". "Hay en la villa unos 600 vecinos; antes hubo más y se marcharon a pelear con los moros del reino de Granada, donde se establecieron con sus mujeres...". "Por Yecla pasan los que de Castilla van a Valencia y a otros puertos de Alicante y Cartagena [...] Los Reyes Católicos concedieron a la villa un mercado franco los martes de cada semana". Yecla (Murcia), 4, 5, 6, 38, 39, 55.

- "Es camino pasajero para Aragón, Navarra, y otros partes". "Tierra de pan llevar y de vino". “... muchos peces”. Jadraque (Guadalajara), 5, 21, 26. 
- “...Produce vino y algún aceite”. "Llega a 400 vecinos con algunos cristianos nuevos". "Por la villa pasan los que van de Toledo a Montiel, a Murcia y a Cartagena”. Villa de Don Fabrique (Toledo), 21, 39, 55.

4.4. Respuestas vinculadas a la tierra o a la propiedad ${ }^{19}$

a) Calidad de la tierra

- “...en la dicha ribera del rio Tajo tienen vecinos de esta Villa plantado las viñas, las cuales son viñas fértiles e de mucho llevar...”. Sayatón (Guadalajara), 21.

b) Justificación a la baja calidad de la tierra

- “...es tierra misera...”. Aldeanueva (Toledo), 26.

- “...por ser áspera la tierra, se coge poco vino...”. Valenzuela de Calatrava (Ciudad Real), 26.

- “...se coge...poco vino por ser montosa...”. Hontanarejo (Ciudad Real), 26.

- “...aunque es buena tierra para ello, son pobres...”. Puebla del Príncipe (Ciudad Real), 26.

c) Justificación a la escasez del término e imposibilidad de roturar nuevas tierras:

- “...el término de esta villa es muy poco...”. Pesadilla (Madrid), 26.

- “...es un término muy chico...”. Irueste (Guadalajara), 26.

- “...es tierra de poco termino...para labores, con veredas que ocupan para ganados herbajeros que pasan...". Iniesta (Cuenca), 26.

- “...poco término tiene este lugar...”. Iriepal (Guadalajara), 26.

- "...traen muy cansada las labores y no hay donde ensanchallas porque no hay donde romper de nuevo tierras ningunas...". Daimiel (Ciudad Real), 26.

d) Tierras en manos de forasteros:

- “...la cosecha deste lugar es vino de vecinos y herederos de Toledo, que tienen aquí sus haciendas...”. Argés (Toledo), 26.

${ }^{19}$ CAMPOS Y FERNÁNDEZ DE SEVILLA, F. J., «La vid y el vino en Castilla La Nueva según las Relaciones topográficas de Felipe II», en Cuadernos de Estudios Manchegos, 27 (2003) 39-63. 
- "...de seis partes de pan y vino, que los vecinos de la dicha villa cogen más de las cinco, se coge en término de la villa de Alarcón...". Quintanar del Rey (Cuenca), 26.

- “...lo mejor son de los vecinos de la villa de Yepes, que lo han comprado de los vecinos de este lugar...". Ciruelos (Toledo), 26.

- “...los diezmos del vino son de vecinos de Toledo...". Villaminaya (Toledo), 26.

- “...la villa de Ajofrín e lugar de Sonseca los vecinos dellos tienen en el dicho termino $[. .$.$] muchas heredades de viñas y tierras...". Mazarambroz$ (Toledo), 26.

Como puede verse en estas respuestas, el vino ya tenía una gran importancia en el siglo XVI. Hoy en día, en el caso español, se cuenta con una gran variedad de fuentes históricas de este tipo, que pueden ayudar a dar un arraigo territorial e histórico a un producto como el vino. Esto las convierte en una valiosa herramienta para el marketing, contribuyendo a la generación de esas experiencias que se han comentado antes. Como referente de puesta en valor territorial se comenta a continuación el ejemplo del Bierzo.

\section{EL BIERZO: PARADIGMA DE MARKETING TERRITORIAL}

El vino y el turismo se han convertido en dos de los principales motores económicos de la comarca del Bierzo. En el ámbito vinícola se ha trabajado en la diferenciación respecto a otras zonas productoras de vino. Una de las claves de la diferenciación del Bierzo es que la variedad autóctona predominante, la Mencía, solamente se encuentra allí y en una zona muy pequeña de Galicia. Se cree que su origen es el Bierzo. Las guías especializadas Peñin y Parker otorgan una puntuación muy alta (superior a 90 sobre 100) a este vino berciano precisamente por tener unas características únicas.

A diferencia de lo que se hace en muchos sitios, en el Bierzo no se ha optado por la homogeneización del sabor del vino, y alejándose de patrones de clasificación de vinos tradicionales, basados en la duración del periodo de envejecimiento (vino crianza, reserva, etcétera), han desarrollado modelos de puesta en valor mediante la trazabilidad y lo autóctono. Estando en sintonía con las preferencias de los nuevos consumidores de vino. Del mismo modo, como estrategia de comunicación, se favorece la interactividad entre bodega y cliente mediante el uso eficiente de las redes sociales y propiciando así un intercambio de información muy valioso. De tal manera que la bodega se beneficia de la promoción de todas sus actividades (incluso venta online), y 
el consumidor puede seguir todo lo que acontece en torno a esa zona, bodega o vino favorito.

\subsection{Inversiones de iniciativa privada entorno al vino en la comarca}

En el Bierzo se encuentran ejemplos muy claros de la utilización del arraigo del vino como reclamo turístico y para promocionar la venta del mismo. Destacan los casos del Palacio de Canedo (Prada a Tope), que se ha convertido en una referencia nacional, y de las nuevas instalaciones en Corullón, donde se ha invertido en viñas situadas en un entorno paisajístico de gran valor. Además, se están llevando a cabo otras iniciativas de importancia en Valtuille de Abajo vinculadas al bodeguero y enólogo Raúl Pérez, y otros.

Todo esto ha llevado a que la región sea considerada una zona virgen, con un clima y una geología muy específicas, propiciando producciones menores, pero de calidad alta. Además, presenta en sus parajes la mayor proporción de viñas centenarias del mundo.

\subsection{Enólogos de prestigio}

Además de atraer inversores, la región también es atractiva para enólogos de prestigio internacional. Estos enólogos trabajan en la diferenciación como apunta el enólogo berciano José Antonio García, buscando conseguir una elaboración más tranquila y centrada en lograr matices diferenciadores. Para ello se está volviendo a las raíces y a la esencia que se ha perdido en otras regiones donde los vinos son más homogéneos. En el Bierzo se están fijando en lo que se está haciendo en otros países, en zonas de prestigio como la Borgoña en Francia o Piamonte en Italia ${ }^{20}$.

Todo este caldo de cultivo, inversiones, e interés científico, ha llevado a varias universidades a interesarse por el vino del Bierzo. Por ejemplo, la Universidad de León está colaborando con algunas bodegas para localizar levaduras propias de la zona y así aumentar las características organolépticas diferenciadoras de ciertos vinos bercianos.

\subsection{El turismo y la promoción en torno al vino}

Investigadores de la Universidad de Vigo llevaron a cabo un estudio en los establecimientos adheridos a la Ruta del Vino del Bierzo para conocer la

\footnotetext{
${ }^{20}$ GARCÍA, J.A., Entrevista personal realizada en Valtuille de Abajo (León), 5 de julio 2019.
} 
oferta de las actividades enoturísticas, el perfil de los enoturistas y la imagen percibida del destino ${ }^{21}$. Según el cronista berciano Prieto el interés y el consumo del vino han aumentado desde que empezó a haber calidad y que, junto al turismo, el vino y todo lo que lo rodea se han convertido en uno de los principales motores económicos del Bierzo. Cuando fue presidente de enoturismo Bierzo se empezaron a llevar a cabo diversas acciones de enoturismo con la cultura del vino como eje central, con actividades de visitas a bodegas y a las viñas. Así mismo se ofrecieron cursos de inglés para los viticultores, señalización de bodegas adheridas, etcétera. Tanto la D.O. Bierzo como los bodegueros unen sus esfuerzos en promocionar los vinos bercianos que tan buena reputación están cosechando últimamente a nivel internacional ${ }^{22}$.

El vino del Bierzo se comercializa en su mayor parte fuera de la comarca y el gran mercado se establece en las exportaciones. Siendo el vínculo turista extranjero, elaboración de vino, comercialización del mismo muy grande, y constituyendo esta forma de proceder en una manera de promocionar el vino en origen.

\subsection{El vino: elemento de fijación de población}

El crecimiento económico ligado al vino lleva, necesariamente, a la fijación de población. Para que haya vino, deben cuidarse las viñas todo el año, algo que es todavía más importante si se recurre a una producción natural, huyendo de la industrialización y manteniendo la esencia tradicional. El trabajo es continuo, primero en la viña, y después en la bodega. Por tanto, la puesta en valor del territorio lo hace más atractivo a las empresas que necesitarán atraer y retener personal especializado en gestión de bodega y comercio internacional.

\subsection{Denominación de origen de vino Bierzo: claves en la internacionalización}

La D.O. Bierzo es una denominación de origen relativamente joven. Está compuesta por 79 bodegas en su mayor parte de pequeño y mediano tamaño. El segmento de precio al que se dirigen es medio-alto. Este año se ha convertido en la primera denominación de origen de España en clasificar todos sus viñedos por unidades geográficas menores dentro de la propia Denominación de Origen ${ }^{23}$.

\footnotetext{
${ }^{21}$ VARIOS, «Turismo enológico y la ruta del vino del Bierzo», en Redmarka. Revista académica de marketing aplicado, 11 (2013) 185-212.

${ }^{22}$ PRIETO, L.M., Entrevista personal realizada en Tejeira (León), 18 de noviembre de 2019.

${ }^{23}$ BOE núm. 201, de 22 de agosto 2019. https://www.boe.es/diario_boe/txt.php?id=BOEB-2019-35528.
} 
El gran patrimonio del Bierzo es el viñedo que tiene. Por superficie porcentual es la mayor concentración de viñedo más viejo del mundo. Esto se debe a sus condiciones tanto geográficas como climatológicas.

\subsection{La trazabilidad y lo autóctono}

La creación de unidades geográficas menores implica zonificar. Esto se ha hecho para diferenciar esas zonas concretas unas de otras en base a su geografía. Estas zonas tienen unos suelos con características concretas, diferentes orientaciones, altitudes y microclimas que hace que exista esta variedad de zonas.

Según la presidenta del Consejo Regulador de la Denominación de Origen Bierzo, la diferenciación de las distintas zonas no quiere decir que haya zonas ni mejores ni peores, simplemente diferentes. Zonas que hacen que los vinos tengan unos parámetros únicos y existan diferencias entre ellas en cuanto a producción, número de años, etcétera. Esto permite al consumidor internacional tener información, a través de la trazabilidad y del etiquetado, de donde viene el vino, municipio, paraje o incluso de qué parcela ${ }^{24}$. Esto se hace en prestigiosas regiones vinícolas en Italia (Piamonte) y en Francia (Borgoña) con la consiguiente repercusión en el precio y posicionamiento que es lo que se busca.

Clasificar viñedos no es tarea fácil, pero es una herramienta que diferencia y da prestigio. Un tercio de las bodegas del Bierzo ya han empezado el proceso de zonificación para identificar el municipio y paraje de procedencia del vino. Por otro lado, la población local ha empezado a proteger las variedades autóctonas al ser un valor añadido y diferenciador.

\section{CONCLUSIONES}

A día de hoy, España es el mayor exportador mundial de vino en volumen. Sin embargo, a diferencia de otros países con los que compite directamente (Francia e Italia), el precio medio del vino español se ha estancado los últimos veinte años. Además, la exportación se centra fundamentalmente en Europa, siendo necesaria una mayor diversificación.

El problema más generalizado a la hora de exportar el vino es la carencia, por parte de cada bodega, de un plan de internacionalización claro y bien

\footnotetext{
${ }^{24}$ BELLO, M., Entrevista personal realizada en Cacabelos (León), 15 de noviembre de 2019.
} 
definido. Además, también es un problema, de cara al precio, la falta de un valor añadido en muchos de los vinos españoles. Los consumidores internacionales no parecen conformarse simplemente con un buen vino, exigen saborear una experiencia, una historia, un territorio, una cultura, etcétera.

La historia se presenta como un elemento que puede servir de eje para la generación de ese valor añadido. Hemos expuesto parcialmente una fuente que se puede utilizar de base para dicho proceso: Las Relaciones de Felipe II. A partir de esa raíz histórica es relativamente fácil ligar el vino a un territorio, a su geografía y a su cultura, aportando el valor añadido que tanto aprecia el consumidor internacional en vinos de calidad.

Finalmente, consideramos que los proyectos que se están llevando a cabo en la comarca del Bierzo son de sumo interés, especialmente la zonificación. Allí se ha desarrollado de manera importante la idea de darle al vino un valor añadido por medio de la singularidad del territorio, de cada municipio, paraje o parcela.

En definitiva, el vino español podría encontrar una mejor aceptación a nivel internacional (y subir su precio) si se le aporta este valor añadido a partir de la relación del vino con el arraigo territorial. Abriéndose de este modo una futura línea de investigación sobre los determinantes territoriales en el éxito exportador de las pymes españolas, y no solo del vino, sino de otros productos agroalimentarios españoles con identificación de origen.

\section{BIBLIOGRAFÍA}

- CAMPOS Y FERNÁNDEZ DE SEVILLA, F. J., «La vid y el vino en Castilla La Nueva según las Relaciones topográficas de Felipe II», en Cuadernos de Estudios Manchegos, 27 (2003) 39-63.

- CAMPOS Y FERNÁNDEZ DE SEVILLA, F. J., «Las Relaciones topográficas de Felipe II: índices, fuentes y bibliografía». Separata del Anuario Jurídico y Económico Escurialense, San Lorenzo del Escorial 2003.

- CAMPOS Y FERNÁNDEZ DE SEVILLA, F. J., «La Mentalidad en Castilla la Nueva en el siglo XVI. Religión, Economía y Sociedad, según las "Relaciones Topográficas”e3 de Felipe II», San Lorenzo del Escorial 1986.

- CAMPOS Y FERNÁNDEZ DE SEVILlA, F. J., Los pueblos de Ciudad Real en las Relaciones Topográficas de Felipe II, Ciudad Real, Diputación de Ciudad Real 2009, 2 vols. 
- CARNICER SOSPEDRA, D., «Cómo vender en el exterion», Valencia, Centros Europeos de Empresas Innovadoras de la Comunidad Valenciana (CEEI CV), 2014.

- CARNICER SOSPEDRA, D., «Plan de internacionalización de empresas», Valencia, Centros Europeos de Empresas Innovadoras de la Comunidad Valenciana (CEEI CV), 2008.

- CARNICER SOSPEDRA, D., «Price in international marketing», Madrid, ESIC Editorial, 2016.

- DEL REY SALGADO, R., «Retos de futuro del sector vitivinícola en España», en La economía del vino en España y en el mundo, 2014. pp. 667-710.

- EUROPEAN COMMISSION, «EU Agricultural Outlook for the agricultural markets and income 2017-2030. »2017. 10 de noviembre de 2019. $<$ https://ec.europa.eu/agriculture/sites/agriculture/files/markets-andprices/medium-term-outlook/2017/2017-fullrep_en.pdf>.

- ICEX, La Economía Internacional de Castilla-La Mancha, 2018-2019. Dirección Territorial de Comercio / ICEX en Castilla-La Mancha Secretaría de Estado de Comercio, Toledo 2019, 27 de junio de 2019. <www.icex.es>.

- OBSERVATORIO ESPAÑOL DEL MERCADO DEL VINO, «10 años de evolución del vino en el mundo y en España.» Observatorio Español del Mercado del Vino. 19 de mayo de 2019. <https://www.oemv.es/dossierestadistico-del-vino-10-aniversario-del-oemv>.

- OBSERVATORIO ESPAÑOL DEL MERCADO DEL VINO «Principales exportadores mundiales de vino - marzo 2019», 10 de noviembre de 2019. $<$ https://www.oemv.es/principales-exportadores-mundiales-de-vino-marzo-2019>.

- ORTEGA RUBIO, J. Relaciones topográficas de los pueblos de España, Madrid: Sociedad Española de Artes Gráficas, 1918.

- REINA PAZ, M., Marketing: qué, cuándo, cómo y dónde, Editorial Sanz y Torres, 2017, p.123

- SÁNCHEZ HERNÁNDEZ, J. L., «El valor social y territorial del vino en España», La economía del vino en España y en el mundo, 2014, pp. 31-66.

- VARIOS, «Turismo enológico y la ruta del vino del Bierzo», en Redmarka. Revista académica de marketing aplicado, 11 (2013) 185-212. 


\section{ANEXO 1. CUESTIONARIO DE LAS RELACIONES}

a) Interrogatorio (cuestiones) de 27 de octubre de $1575^{25}$.

1. «Primeramente, se declare y diga el nombre del pueblo cuya relación se hiciese; cómo se llama al presente y por qué se llaman así. Y si se ha llamado de otra manera antes de ahora; y también por qué se llamó así, si se supiere.

2. Si el dicho pueblo es antiguo o nuevo, y desde qué tiempo acá está fundado, y quién fue el fundador, y cuando se ganó de los moros, o lo que de ello se supiese.

3. Si es ciudad, villa, o aldea; y si fuese ciudad o villa, desde qué tiempo acá lo es, y el título que tiene: y si fuese aldea, en que jurisdicción de ciudad o villa cae.

4. El reino en que comúnmente se cuenta el dicho pueblo, como es decir si cae en el reino de Castilla, o de León, Galicia, Toledo, Granada, Murcia, Aragón, Valencia, Cataluña, o Navarra, y en qué provincia o comarca de ellos, como sería decir en tierra de Campos, Rioja, Alcarria, la Mancha, etc.

5. Y si el pueblo que está en frontera de algún reino extraño, qué tan lejos está de la raya, y si es entrada o paso para él, [En letra del tiempo se añade: o puerto, o aduana do se cobran algunos derechos].

6. El escudo de armas que el dicho pueblo tuviese, si tuviese algunas, y por qué causa o razón las ha tomado, si se supiese algo.

7. El señor dueño del pueblo, si es del Rey, o de algún señor particular, o de alguna de las Ordenes de Santiago, Calatrava, Alcántara, o San Juan, o si es behetría, y por qué causa, y cuándo se enajenó de la corona real y vino a ser cuyo fuese, si de ello se tuviere noticia.

8. Si el pueblo de quién se hiciese relación fuese ciudad o villa, se declare si tiene voto en Cortes; y si no, que ciudad o villa habla por él, o a donde acude para las juntas o concejos o repartimientos que se hiciesen.

9. La chancillería en cuyo distrito cae el tal pueblo, y a dónde van los pleitos en grado de apelación, y las leguas que hay desde el dicho pueblo hasta donde reside la dicha chancillería.

10. La gobernación, corregimiento, alcaldía, merindad o adelantamiento en que está el dicho pueblo; y si fuere aldea, cuántas leguas hay hasta la ciudad o villa de cuya jurisdicción fuese.

11. Ítem, el Arzobispado, o Obispado, o Abadía y Arciprestazgo en que cae el dicho pueblo, cuya relación se hiciese, y las leguas que hay hasta el pueblo donde reside la catedral, o que es cabecera de su partido.

12. Si fuere alguna de las Ordenes de Santiago, Calatrava, Alcántara o San Juan, se diga el Priorato y partido de ellas, en que cayese el dicho pueblo.

${ }^{25}$ CAMPOS Y FERNÁNDEZ DE SEVILLA, F. J., «Las Relaciones topográficas de Felipe II: índices, fuentes y bibliografia», Separata del Anuario Jurídico y Económico Escurialense, San Lorenzo del Escorial, 2003. 
13. Assi mesmo se diga el nombre del primer pueblo que hubiese, yendo del lugar donde se hiciese la dicha relación, hacia donde el sol sale, y las leguas que hasta él hubiese, declarando poco más o menos si el dicho pueblo está directamente hacia donde el sol sale, o desviado algo al parecer, y a qué mano; y si las leguas son ordinarias, grandes o pequeñas, y por camino derecho o por algún rodeo.

14. Item, se diga el nombre del primer pueblo que hubiese, yendo de donde se hiciese la relación hacia el medio día, y el número de las leguas que hubiese, y si son grandes o pequeñas, o por camino derecho o torcido, y si el tal pueblo está derecho al medio día, o al parecer algo desviado, y a qué parte.

15. Y assi mesmo, se declare el nombre del primer pueblo que hubiese caminado para el poniente desde el dicho pueblo, con él número de las leguas que hay hasta él, y si son grandes o pequeñas, y por camino derecho o no; y si está derecho al poniente o no; como queda dicho en los capítulos anteriores de este.

16. Y otro tanto se dirá del primer pueblo que hubiese a la parte del norte o cierzo, diciendo el nombre de él, y las leguas que hay hasta el pueblo donde se hace la relación; y si son grandes o pequeñas, y por camino derecho, y si el pueblo está derecho al norte o no; todo como queda dicho en los capítulos precedentes.

17. La calidad de la tierra en que está el dicho pueblo, si es tierra caliente o fría, tierra llana o serranía, rasa o montañosa y áspera, tierra sana o enferma.

18. Si es tierra abundosa, o falta de leña, y de donde se proveen; y si montañosa, de qué monte y arboleda, y qué animales, cazas y salvaginas se crían y hallan en ella.

19. Si estuviese en serranía el pueblo, cómo se llaman las sierras en que esté, o que estuvieren cerca de él y cuánto está apartado de ellas, y a qué parte le caen, y de dónde vienen corriendo las dichas sierras, y adónde van aparar.

20. Los nombres de los ríos que pasaren por dicho pueblo, o cerca de él, y qué tan lejos, y a qué parte de él pasan, y cuán grandes y caudalosos son.

21. Las riberas, huertas, regadíos y las frutas, y otras cosas que en ellas se cogen, y los pescados y pesquerías que los dichos ríos hubiere, y los dueños y señores de ellos, y lo que les suele valer y rentar.

22. Los molinos y aceñas, y los barcos y puentes señalados que en los dichos ríos y términos del dicho lugar hubiese, y los aprovechamientos de ellos, y cuyos son.

23. Si es abundoso o falto de aguas, y las fuentes o lagunas señaladas que en el dicho pueblo y sus términos hubiese; y si no hay ríos ni fuentes, de dónde beben y a dónde van a moler.

24. Los pastos y dehesas señaladas que en términos del sobredicho pueblo hubiese, con los bosques y cotos de caza y pesca que asimismo hubiese, y cuyos son y lo que valen.

25. Las casas de encomiendas, cortijos y otras haciendas señaladas que hubiese en tierra del dicho pueblo, públicas o de particulares.

26. Y si es tierra de labranza, las cosas que en ella más cogen y dan, y los ganados que se crían y hay, y lo que comúnmente suele cogerse de los diezmos, y lo que valen, y las cosas de que tienen más falta, y de dónde se proveen de ellas.

27. Si hay minas de oro, plata, hierro, cobre, plomo, azogue, y otros metales y minerales de tinturas y colores.

28. Las salinas que en tierra de dicho pueblo hay, y las canteras de jaspes, mármol y otras piedras estimadas que se hallaren en ella. 
29. Y si el pueblo fuese marítimo, qué tan lejos o cerca está de la mar, y la suerte de la costa que alcanza, si es costa brava o baja, y los pescados que se pescan en ella.

30. Los puertos, bayas y desembarcaderos que hubiese en la costa de la dicha tierra, con las medidas del ancho y largo de ellas, y relación de las entradas, y fondo, y seguridad que tienen, y la provisión de agua y leña que alcanzan.

31. La defensa de fortalezas que hubiese en los dichos puertos para seguridad de ello, y los muelles y atarazanas que hubiese.

32. El sitio y asiento donde el dicho pueblo está poblado; si está en alto o en bajo, llano o áspero; y si es cercado, las cercas y murallas que tiene y de qué son.

33. Los castillos, torres y fortalezas que en el pueblo y jurisdicción de él hubiere, y la fábrica y materiales de que son, con relación de las armas y municiones que en ellas hubiese.

34. Los alcaides de las fortalezas y castillos, y quien los posee, y lo que valen las alcaldías, sus salarios y aprovechamientos, y las preeminencias que tuviesen.

35. Las suertes de las casas y edificios que se usan en el pueblo, y de qué materiales están edificadas, y si los materiales los hay en la tierra o los traen de otra parte.

36. Los edificios señalados que en el pueblo hubiese, y los rastros de edificios antiguos, epitafios y letreros, y antiguallas de que hubiese noticia.

37. Los pueblos señalados y cosas dignas de memoria, de bien o mal, que hubiesen acaecido en dicho pueblo o en sus términos, y los campos, montes y otros lugares nombrados por algunas batallas, robos o muertes, y otras cosas notables que en ellos haya habido.

38. Las personas señaladas en letras o armas, o en otras buenas o malas que haya en el dicho pueblo, o hayan nascido o salido de él, con lo que supiese de sus hechos y dichos, y otros cuentos graciosos que en los dichos pueblos haya habido.

39. Las casas y [Ms. número de] vecinos que al presente en el dicho pueblo hubiese, y si ha tenido más o menos antes de ahora, y la causa por que se haya disminuido.

40. Si los vecinos con todos labradores, o parte de ellos hidalgos, y el número de los hijosdalgo que hay, y de qué privilegios y exenciones gozan.

41. Los mayoradgos que hay en el dicho pueblo, y las casas y solares de linages que hay en él, si ello se alcanzase a saber algo.

42. Si la gente del dicho pueblo es rica o pobre, las granjerías, tratos y oficios de que viven, y las cosas que allí se hacen, o se han labrado, o labran mejor que en otras partes.

43. Las justicias eclesiásticas o seglares que hay en dicho pueblo y quién las posee; y si en el gobierno y administración de justicia hubiese alguna diferencia de lo que en otras partes se platica.

44. Los ministros de justicia eclesiástica y seglar que hubiese en el dicho pueblo, y el número de regidores, alguaciles y escribanos, y otros oficios y oficiales de concejo, y los salarios y aprovechamientos de cada uno tuviese.

45. Los términos propios que el dicho pueblo tiene, y los comunes y realengos de que goza, y las rentas y aprovechamiento que tiene por propios del dicho pueblo, y lo que valen [Ms. los portazgos y pasages del].

46. Los privilegios, fueros y costumbres notables que el tal pueblo tiene y hubiera tenido, y la razón por qué se le dieron, si se supiere, y los que se le guardan y han dejado de guardar, y por qué no se le guardan ya, y desde qué tiempo acá.

47. Si el pueblo es de señorío se diga si la jurisdicción es de señor o no, y las rentas y aprovechamientos, y los privilegios y preeminencias que dichos señores o algunas otras personas particulares tuviesen en dicho pueblo. 
48. La iglesia catedral, o colegial, que hubiese en el dicho pueblo, y las parroquias que hubiese, con alguna breve relación de las capillas y enterramientos, y donaciones señaladas que en ellas haya [Ms. y la vocación dellas].

49. Las prebendas, calongias y dignidades que en la catedral y colegial hubiere, con alguna relación de lo que valen.

50. Y los arciprestazgos, beneficios curados y simples, con sus anejos y préstamos, que hubiese en las iglesias parroquiales, y lo que valen.

51. Las reliquias notables que en las dichas iglesias y pueblos hubiere; y las hermitas señaladas, y devocionarios de su jurisdicción; y los milagros que en él se hubiesen hecho.

52. Las fiestas de guardar, y días de ayuno, y de no comer carne, que en el pueblo se guardasen por voto por (sic) particular, demás de las de la Iglesia, y las causas y principio de ellas.

53. Los monasterios de frayles, monjas y beatas que hubiese en el pueblo y su tierra, con lo que se supiese de sus fundadores, y el número de religiosos y rentas que hubiere.

54. Los hospitales y obras pías que hay en el dicho pueblo, y las rentas que tienen, y lo que valen, con los instituidores de ellas.

55. Si el pueblo fuere pasagero, en qué camino real estuviese, y las rentas que hubiere en la tierra y términos de él, y cuyas son, y lo que valen.

56. Los sitios de los pueblos y lugares despoblados que hubiese en la tierra, y el nombre que tuvieron, y la causa por qué se despoblaron.

57. Y generalmente, todas las demás cosas notables y dignas de saberse que se ofreciesen, a propósito para la historia y descripción del sobre dicho pueblo, aunque no vayan apuntadas, ni escritas en esta memoria. [Ms. y los anexos que el dicho pueblo tuviese y quántas leguas del está, y si son concejo por si, o no. El número de los vs. (vecinos) y las otras cosas dellas conforme a esta memoria].

[Siguen estos seis renglones manuscritos de letra que consideramos, como la anterior, de Antonio Gracián, secretario de Felipe II].

[58] Item en la Relación de cada pueblo se digan los nombres de los pueblos de señorío, o de órdenes que tuviese junto dél en sus contornos, y cuyos son, y el número de vezínos que tuvieren, poco más o menos, con alguna particularidad notable dellas, si se supiere.

[59] Las ferias y mercados de dicho pueblo, que tan grandes y caudalosos son; y si son francos en todo, o en algunas cosas; los días dellas en que se hacen, quiénes se las concedió, y desde qué tiempo acá, y por qué privilegios"». 